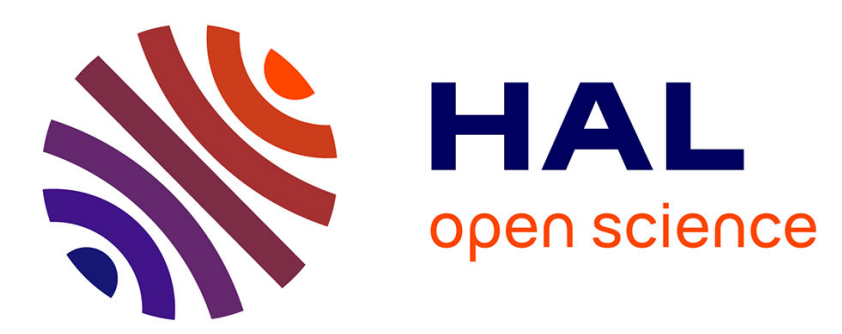

\title{
A New Integral Formulation for Eddy Current Computation in Thin Conductive Shells
}

Tung Le Duc, Gérard Meunier, Olivier Chadebec, Jean-Michel Guichon

\section{To cite this version:}

Tung Le Duc, Gérard Meunier, Olivier Chadebec, Jean-Michel Guichon. A New Integral Formulation for Eddy Current Computation in Thin Conductive Shells. IEEE Transactions on Magnetics, 2012, 48 (2), pp.427-430. 10.1109/TMAG.2011.2173920 . hal-00727820

\section{HAL Id: hal-00727820 \\ https://hal.science/hal-00727820}

Submitted on 4 Sep 2012

HAL is a multi-disciplinary open access archive for the deposit and dissemination of scientific research documents, whether they are published or not. The documents may come from teaching and research institutions in France or abroad, or from public or private research centers.
L'archive ouverte pluridisciplinaire HAL, est destinée au dépôt et à la diffusion de documents scientifiques de niveau recherche, publiés ou non, émanant des établissements d'enseignement et de recherche français ou étrangers, des laboratoires publics ou privés. 


\title{
A New Integral Formulation for Eddy Current Computation in Thin Conductive Shells
}

\author{
T. Le-Duc, G. Meunier, O. Chadebec, and J.-M. Guichon \\ Grenoble Electrical Engineering Laboratory, University of Grenoble, Grenoble-INP/Université Joseph Fourier, \\ CNRS UMR 5269, Grenoble, France
}

\begin{abstract}
In order to compute eddy current distributions in thin conductive nonmagnetic shells, a new integral formulation is proposed. The method is based on a surface impedance condition which takes into account the field variation through depth due to skin effect. It is general and enables the modeling of various problems whatever their skin-depth and avoiding the meshing of the air region.
\end{abstract}

Index Terms-Integral equation method, quasi-static problems, surface impedance condition, thin conductive shells.

\section{INTRODUCTION}

I NTEGRAL formulations dedicated to the computation of eddy currents in thin shells for the case of a skin depth $\delta$ much greater than the thickness $e(\delta \gg e)$ has already been proposed by many authors [1]-[5]. In this case, the eddy current distribution is supposed to be uniform across the thickness and surface elements are used avoiding the meshing of the shell's thickness. Such formulations are currently well-known and have shown good accuracy with a few numbers of elements in comparison with finite element methods where the air region needs to be meshed.

However, the computation of eddy currents in thin shells in the general case ( $\delta \ll e$ or $\delta \approx e$ for instance) is still a difficulty and has only been studied by few authors.

In [6], a general shell element formulation has been proposed. Based on a pseudo-analytical solution (solution of the 1D problem) and a nodal approximation of the scalar magnetic potential, the field variation across the thickness of the shell (i.e., the skin depth) is taken into account with a quite good accuracy. In this formulation, the air region is modeled by boundary integral equations and the coupling is realized at the interface on both sides of the shell.

In [7], a very similar shell element for modeling thin conducting regions has been proposed. Like in [6], this element takes into account the field variation through depth due to skin effect but in this paper the outside regions are modeled with finite elements method. However, the approach presented in [7] leads to the mesh of the air region. If shielding devices are modeled, this task is complex and can lead to inaccuracies.

This paper presents a new integral formulation which allows the modeling of nonmagnetic conductive thin shells in the case general $(\delta \gg e$ or $\delta \approx e$ or $\delta \ll e)$. Based on a simple surface discretization, the number of unknowns considerably reduces, the depth of the shell being not meshed. Moreover, like in [6], it does not require the meshing of the air region. An advantage of our new formulation in comparison with existing integral ones is its compactness and its generality. Indeed, it can be applied

Manuscript received July 06, 2011; revised September 27, 2011; accepted October 18, 2011. Date of current version January 25, 2012. Corresponding author: O. Chadebec (e-mail: Olivier.Chadebec@g2elab.grenoble-inp.fr)

Color versions of one or more of the figures in this paper are available online at http://ieeexplore.ieee.org.

Digital Object Identifier 10.1109/TMAG.2011.2173920

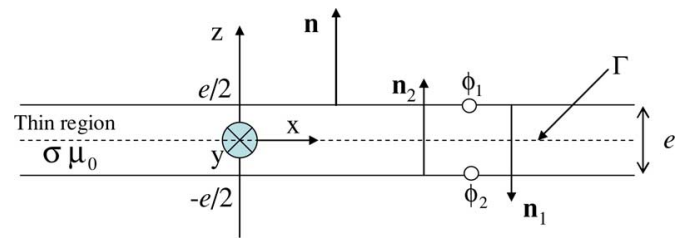

Fig. 1. Thin conductive and nonmagnetic region and associated notations.

easily to every shell geometry, either delimited by closed surface or not. Moreover, we will see that the formulation is less consuming in terms of degrees of freedom than [6].

In the second part of the paper, a surface impedance condition for the shell will be presented. In the third part, this surface condition will be coupled with an integro-differential approach. Finally, two numerical examples will be proposed in the last part. Results obtained with our formulation will be compared by those given by the Finite Element Method (FEM).

\section{SuRfACE IMPEDANCE CONDITION FOR THE SHELL}

We consider a nonmagnetic shell with a thickness $e$ with a skin depth $\delta$ (Fig. 1). Both thin shell and surrounding regions are nonmagnetic $\left(\mu=\mu_{0}\right)$, the conductivity is $\sigma$.

In our approach, the tangential component variation of the magnetic field across the thickness of the shell is approximated by the analytical solution of the problem obtained for an infinite plane. For such problem, we get [6], [7]:

$$
\mathbf{H}_{s}(z)=\frac{1}{\operatorname{sh}(a e)}\left[\mathbf{H}_{1 s} \operatorname{sh}\left(\frac{a e}{2}+a z\right)+\mathbf{H}_{2 s} \operatorname{sh}\left(\frac{a e}{2}-a z\right)\right]
$$

where, $a=(1+j) / \delta$ and $\mathbf{H}_{1 s}$ and $\mathbf{H}_{2 s}$ are the tangential field values on both sides of the shell. The volume eddy curent density being tangential, thus by applying Ampere's law, we get:

$$
\mathbf{J}=\mathbf{c u r l} \mathbf{H}=-\mathbf{n}_{1} \times \frac{\partial \mathbf{H}_{S}(z)}{\partial z}
$$

By introducing (1) in (2), we get:

$\mathbf{J}(z)=-\frac{a}{\operatorname{sh}(a e)} \mathbf{n}_{1} \times\left[\mathbf{H}_{1 s} \operatorname{ch}\left(\frac{a e}{2}+a z\right)-\mathbf{H}_{2 s} \operatorname{ch}\left(\frac{a e}{2}-a z\right)\right]$.

Expression of electric field on side " 1 " of the shell is:

$$
\mathbf{E}_{1}=\mathbf{E}(e / 2)=\frac{\mathbf{J}(e / 2)}{\sigma}
$$

Equations (4) and (3) are combined to get:

$$
\mathbf{E}_{1}=\mathbf{n}_{1} \times\left(\beta \mathbf{H}_{2 s}-\alpha \mathbf{H}_{1 s}\right)
$$


with

$$
\alpha=\frac{a}{\sigma \operatorname{th} a e}, \quad \beta=\frac{a}{\sigma \operatorname{sh} a e} .
$$

In a second step, Faraday's law is projected normally to the shell:

$$
\mathbf{n} \cdot \operatorname{curlE}=-\mathbf{n} \cdot j \omega \mathbf{B} .
$$

Applying Galerkin method to (6) on side 1 of the shell surface $\Gamma_{1}$ leads to:

$$
j \omega \int_{\Gamma_{1}} \mathrm{w} \cdot \mathbf{B} \cdot \mathbf{n} \mathrm{d} \Gamma=-\int_{\Gamma_{1}} \mathrm{w}(\mathbf{c u r l E}) \cdot \mathbf{n} \mathrm{d} \Gamma
$$

where $w$ is a set of nodal surface weighting functions, the shell being limited to $\Gamma$.

Applying $\operatorname{curl}(m \mathbf{U})=m \mathbf{c u r l} \mathbf{U}+(\operatorname{grad} m) \times \mathbf{U}$ property in the second term of (7), we get following expression:

$$
\begin{aligned}
j \omega \int_{\Gamma_{1}} \mathrm{w} \cdot \mathbf{B} \cdot \mathbf{n} \mathrm{d} \Gamma=\int_{\Gamma_{1}}\left(\operatorname{grad}_{\mathrm{s}} \mathrm{w} \times \mathbf{E}\right) \cdot \mathbf{n} \mathrm{d} \Gamma \\
\\
\quad-\int_{\Gamma_{1}} \operatorname{curl}(\mathrm{w} \cdot \mathbf{E}) \cdot \mathbf{n} \mathrm{d} \Gamma .
\end{aligned}
$$

We switch terms in the mixed product of the second integral of (8) and apply Stokes theorem to the third one to get:

$$
j \omega \int_{\Gamma_{1}} \mathrm{w} \cdot \mathbf{B} \cdot \mathbf{n} \mathrm{d} \Gamma=\int_{\Gamma_{1}}(\mathbf{E} \times \mathbf{n}) \cdot \operatorname{grad}_{\mathrm{s}} \mathrm{w} \cdot \mathrm{d} \Gamma-\int_{\lambda_{1}} \mathrm{w} \cdot \mathbf{E} \cdot \mathrm{d} \lambda
$$

where $\lambda_{1}$ is the line region delimiting the $\Gamma_{1}$ surface. Using (5) and (9), we finally have:

$$
\begin{aligned}
\int_{\Gamma_{1}} \operatorname{grad}_{\mathrm{s}} \mathrm{w} \cdot( & \left.\alpha \mathbf{H}_{1 \mathrm{~s}}-\beta \mathbf{H}_{2 \mathrm{~s}}\right) \mathrm{d} \Gamma \\
& +j \omega \int_{\Gamma_{1}} \mathrm{w} \cdot \mathbf{B}_{1} \cdot \mathbf{n}_{1} \cdot \mathrm{d} \Gamma+\int_{\lambda_{1}} \mathrm{w} \cdot \mathbf{E} \cdot \mathrm{d} \lambda=0 .
\end{aligned}
$$

On the side 2 of the shell, we get a similar equation:

$$
\begin{aligned}
\int_{\Gamma_{2}} \operatorname{grad}_{\mathrm{s}} \mathrm{w} & \cdot\left(\alpha \mathbf{H}_{2 \mathrm{~s}}-\beta \mathbf{H}_{1 \mathrm{~s}}\right) \mathrm{d} \Gamma \\
& +j \omega \int_{\Gamma_{2}} \mathrm{w} \cdot \mathbf{B}_{2} \cdot \mathbf{n}_{2} \cdot \mathrm{d} \Gamma+\int_{\lambda_{2}} \mathrm{w} \cdot \mathbf{E} \cdot \mathrm{d} \lambda=0 .
\end{aligned}
$$

Equations (10) and (11) represent the electromagnetic behavior of the conductive shell itself. In order to take into account the surrounding region, it remains to couple the two formulations. In [7], the authors choose to use the Finite Element Method (FEM), thus they need to mesh the air region. In [6], a Boundary Equation Method (BEM) is preferred but the authors have to manage the coupling on both sides of the shell and have to determine if both sides of the shell are interfaced with a single region or with two different ones. This leads to the development of quite sophisticated geometrical analysis tools if the method has to be applied to general geometry. Moreover, four unknowns per node are needed. We have preferred a new approach, based on the coupling of (10) and (11) with an integral volume equation. The obtained formulation presents an interesting compactness treating different air volumes as the single physical region. Moreover two unknowns per node are needed (two potentials on both sides of the shell).

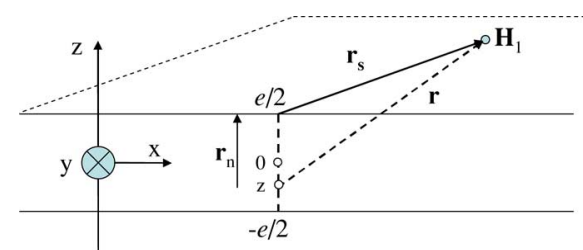

Fig. 2. Magnetic field created by eddy current distribution and associated notations.

\section{VOLUME INTEGRAL FORMULATION}

Let us now assume that the shell is placed in an inductor field $\mathbf{H}_{0}$. On the side "1" of the shell, the total magnetic field $\mathbf{H}_{1}$ is the sum of $\mathbf{H}_{01}$ and $\mathbf{H}_{1 \mathrm{r}}$, the reaction of the eddy current in thin shell. Thanks to the law of Biot and Savart, we can write:

$$
\mathbf{H}_{1}=\mathbf{H}_{0}+(1 / 4 \pi) \int_{\Gamma_{1}} \int_{-\mathrm{e} / 2}^{\mathrm{e} / 2} \mathbf{J}(z) \times\left(\mathbf{r} / \mathrm{r}^{3}\right) \mathrm{d} z \mathrm{~d} \Gamma
$$

where $\mathbf{r}$ is the vector linking the integration point to the point where the field is expressed (Fig. 2). Additional expressions can be given:

$$
\mathbf{r}=\mathbf{r}_{s}-(e / 2-z) \cdot \mathbf{n}_{1} \text { and } r^{3}=\left(r_{s}^{2}+(e / 2-z)^{2}\right)^{3 / 2} .
$$

Using (3), (12) and (13), we get

$$
\mathbf{H}_{1}=\mathbf{H}_{0}+(1 / 4 \pi) \int_{\Gamma_{1}}\left(\mathbf{R}_{1} \cdot \mathbf{H}_{1 \mathrm{~s}}-\mathbf{R}_{2} \cdot \mathbf{H}_{2 \mathrm{~s}}\right) \mathrm{d} \Gamma
$$

with:

$$
\begin{aligned}
\mathbf{R}_{1}= & -\frac{a}{\operatorname{sh}(a e)} \\
& \times \int_{-e / 2}^{e / 2} \frac{c h(a e / 2+a z) \cdot \mathbf{n}_{1} \times\left(\mathbf{r}_{s}-(e / 2-z) \cdot \mathbf{n}_{1}\right)}{\left(r_{s}^{2}+(e / 2-z)^{2}\right)^{3 / 2}} d z \\
\mathbf{R}_{2}= & -\frac{a}{\operatorname{sh}(a e)} \\
& \times \int_{-e / 2}^{e / 2} \frac{\operatorname{ch}(a e / 2-a z) \cdot \mathbf{n}_{1} \times\left(\mathbf{r}_{s}-(e / 2-z) \cdot \mathbf{n}_{1}\right)}{\left(r_{s}^{2}+(e / 2-z)^{2}\right)^{3 / 2}} d z .
\end{aligned}
$$

Using (15), (10) becomes:

$$
\begin{aligned}
& \int_{\Gamma_{1}} \operatorname{grad}_{\mathrm{s}} \mathrm{w} \cdot\left(\alpha \mathbf{H}_{1 \mathrm{~s}}-\beta \mathbf{H}_{2 \mathrm{~s}}\right) \mathrm{d} \Gamma+\int_{\lambda_{1}} \mathrm{w} \cdot \mathbf{E} \cdot \mathrm{d} \lambda \\
& \quad+j \omega \mu_{0} \int_{\Gamma_{1}} \mathrm{w}\left(\mathbf{H}_{0}+\frac{1}{4 \pi} \int_{\Gamma_{1}}\left(\mathbf{R}_{1} \mathbf{H}_{1 \mathrm{~s}}-\mathbf{R}_{2} \mathbf{H}_{2 \mathrm{~s}}\right) \mathrm{d} \Gamma\right) \\
& \quad \cdot \mathbf{n}_{1} \cdot \mathrm{d} \Gamma=0 .
\end{aligned}
$$

A reduced magnetic scalar potential is now introduced for each side of the shell. Since there is a jump of the tangential component of the magnetic field through the element, a double layer node surface element is needed (Fig. 3) [7]. Let us notice that if two potential values are associated to each node, only surface elements are needed in order to discretized the shell. 


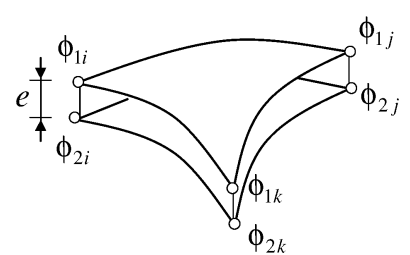

Fig. 3. Shell element representation with associated potential jump.

Expressions of tangential magnetic fields on both sides and outside of the shell are:

$$
\begin{aligned}
& \mathbf{H}_{1 s}=\mathbf{H}_{0 s}-\operatorname{grad}_{s} \phi_{1} \\
& \mathbf{H}_{2 s}=\mathbf{H}_{0 s}-\operatorname{grad}_{s} \phi_{2}
\end{aligned}
$$

where $\mathbf{H}_{0 s}$ is the inductor field limited to its tangential component (same value on both sides of the shell, the thickness being small) and $\phi_{1}, \phi_{2}$ stand for magnetic scalar potentials on both sides. Using (17), (18) and (19), we obtain the final formulation corresponding to the side " 1 " of the shell:

$$
\begin{aligned}
- & \alpha \int_{\Gamma_{1}} \operatorname{grad}_{\mathrm{s}} \mathrm{w} \cdot \operatorname{grad}_{\mathrm{s}} \phi_{1} \cdot \mathrm{d} \Gamma \\
& +\beta \int_{\Gamma_{1}} \operatorname{grad}_{\mathrm{s}} \mathrm{w} \cdot \operatorname{grad}_{\mathrm{s}} \phi_{2} \cdot \mathrm{d} \Gamma+\left(j \omega \mu_{0} / 4 \pi\right) \int_{\Gamma_{1}} \mathrm{w} \\
& \times\left(\int_{\Gamma_{1}}-\mathbf{R}_{1} \operatorname{grad}_{\mathrm{s}} \phi_{1} \mathrm{~d} \Gamma+\int_{\Gamma_{1}} \mathbf{R}_{2} \operatorname{grad}_{\mathrm{s}} \phi_{2} \mathrm{~d} \Gamma\right) \mathrm{d} \Gamma \\
& +\int_{\lambda_{1}} \mathrm{w} \cdot \mathbf{E} \cdot \mathrm{d} \lambda=-\left(j \omega \cdot \mu_{0} / 4 \pi\right) \int_{\Gamma_{1}} \mathrm{w} \\
& \left.\cdot \int_{\Gamma_{1}}\left(\mathbf{R}_{1}-\mathbf{R}_{2}\right) \mathbf{H}_{0 \mathrm{~s}} \mathrm{~d} \Gamma\right] \mathrm{d} \Gamma \quad \int_{\Gamma_{1}} \mathrm{w} \cdot \mathbf{H}_{0} \cdot \mathbf{n}_{1} \mathrm{~d} \Gamma .
\end{aligned}
$$

The equation on the side " 2 " of shell is obtained by permuting the subscripts " 1 " and " 2 ". Both equations are solved simultaneously in order to get both potential $\phi_{1}$ and $\phi_{2}$ distributions. It should be pointed out that on $\lambda$ line, delimiting the external border of the shell, the potential jump is set to zeros (continuity of the potential ensuring that the current is tangential to the edge) so both integral terms $\int_{\lambda} \mathrm{w} \cdot \mathbf{E} \cdot \mathrm{d} \lambda$ vanish so are not taken into account in the final resolution.

These equations have to be discretized. The easiest way is to mesh the average surface $\Gamma$, situated halfway between boundaries of the shells (Fig. 1), into $n$ triangular elements associated with a uniform tangential component of the eddy current (meaning 1-order shape functions for the potential). The obtained algebraic linear system has size $2 p$ (two complex magnetic scalar potentials per node; the mesh is being composed of $p$ nodes). Let us notice that shape functions with higher orders can be used.

It must be pointed out that this formulation looks like a mix between finite element method and integral volume one. In (20), both first terms leads to sparse matrices (similar to which given by a FEM standard integration process) but the last one in-

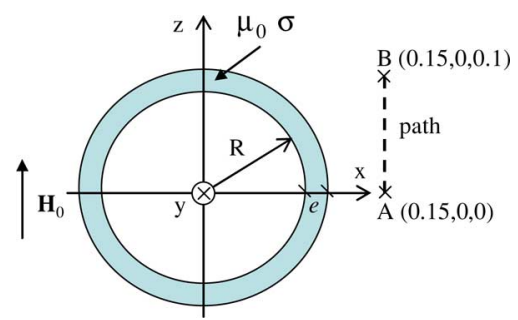

Fig. 4. Hollow sphere, notations and path where the external magnetic field is computed.

volving $R_{1}$ and $R_{2}$ is associated to an integral method. The matrix system obtained is so fully populated and thus the integration time and the memory needed could quickly become prohibitive. Compression algorithms must be used if problems with large numbers of elements are addressed. The use of the well-known «Fast Multipole Method» (FMM) [8], [9] to compress law of Biot and Savart is well-known to be very efficient in such configuration. Moreover, integrals (15) and (16) can be singular if we consider the influence of the element on itself. The integral is computed thank the use of an adaptive quadrature.

\section{NUMERICAL EXAMPLES}

The new formulation has been implemented for a 3-D geometry and has been validated with two different academic examples. A comparison with FEM method using FLUX software [8] is made.

\section{A. Hollow Sphere}

Our formulation is based on the infinite plane assumption for the analytical computation of skin effect across the shell thickness. Even if this assumption has already demonstrated its efficiency, some inaccuracies can appear especially closed to shells edges or corners. Our first validation case deals with geometry without this kind of configuration: A conductive hollow sphere $(\mathrm{R}=0.1 \mathrm{~m}, e=2 \mathrm{~mm}, \sigma=6 \mathrm{E} 7 \mathrm{~S} / \mathrm{m})$ placed in a uniform axial magnetic field $\mathbf{H}_{0}=\left[\begin{array}{lll}0 & 0 & 1\end{array}\right](\mathrm{A} / \mathrm{m})$ (Fig. 4) is considered.

Three configurations have been tested:

- case $1: \mathrm{f}=50 \mathrm{~Hz}$, skin depth $\delta$ greater than the thickness $e(\delta=9.2 \mathrm{~mm}, \delta>e)$

- case 2: $\mathrm{f}=1000 \mathrm{~Hz}$, skin depth is comparable to the thickness $e(\delta=2.1 \mathrm{~mm}, \delta \approx e)$

- case 3: $\mathrm{f}=4000 \mathrm{~Hz}$, skin depth $\delta$ smaller than the thickness $e(\delta=1 \mathrm{~mm}, \delta<e)$

For each case, eddy current losses (Joule losses) in the sphere have been calculated and compared with results given by axisymmetric FEM (Table I). The shell has been meshed with volume elements. In a second step, we have computed the magnetic field in the air region close to the device (calculated on the path $\mathrm{AB}$ in Fig. 3) with both methods for two cases: $\mathrm{f}=50 \mathrm{~Hz}$ and $f=4000 \mathrm{~Hz}$ (Fig. 5).

We can see small differences between computed values with our formulation and the reference (axisymmetric FEM). These differences can be explained by the infinite plane assumption and certainly by the use of a 1-order triangular coarse mesh. A good solution would be to refine it but matrix compression algorithms have not been implemented yet.

\section{B. Thin Conductive Disk}

The second test case is a thin conductive disk $(\mathrm{R}=1 \mathrm{~m}$, $e=20 \mathrm{E}-3 \mathrm{~m}, \sigma=6 \mathrm{E} 7 \mathrm{~S} / \mathrm{m}$ ) placed in a magnetic field 


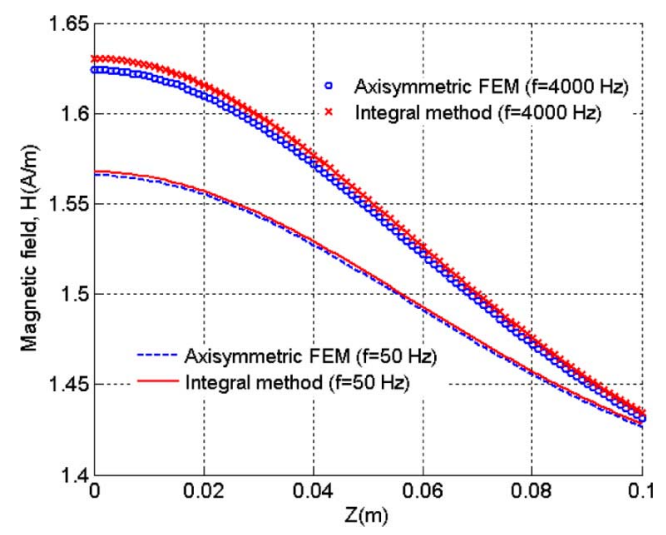

Fig. 5. Magnetic field in the path $\mathrm{AB}$ calculated by 2 methods with $\mathrm{f}=50 \mathrm{~Hz}$ and $\mathrm{f}=4000 \mathrm{~Hz}$.

TABLE I

JOULE LOSSES OF THE HOLLOW SPHERE

\begin{tabular}{|c|l|l|l|}
\hline$e / \delta$ & $\begin{array}{c}\text { Loss Joule (W) } \\
\text { computed by our } \\
\text { method }\end{array}$ & $\begin{array}{c}\text { Loss Joule (W) } \\
\text { computed by } \\
\text { axisymmetric FEM }\end{array}$ & Diff. \\
\hline 0.22 & $1.16 \mathrm{E}-6$ & $1.14 \mathrm{E}-6$ & $1.74 \%$ \\
\hline 0.97 & $1.75 \mathrm{E}-6$ & $1.71 \mathrm{E}-6$ & $2.34 \%$ \\
\hline 2.00 & $2.79 \mathrm{E}-6$ & $2.90 \mathrm{E}-6$ & $3.71 \%$ \\
\hline
\end{tabular}

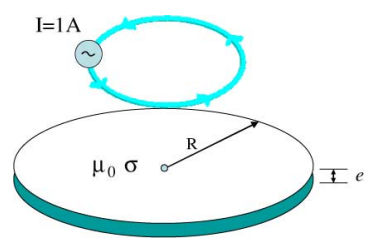

Fig. 6. Thin conductive disk, exciting coil and notations. Disk characteristics: $\mathrm{R}=1 \mathrm{~m}, e=20 \mathrm{E}-3 \mathrm{~m}, \sigma=6 \mathrm{E} 7 \mathrm{~S} / \mathrm{m}$. Coils characteristics: $\mathrm{R}_{\text {coil }}=0.5$ $\mathrm{m}$, located $1 \mathrm{~m}$ high above the disk, $\mathrm{I}=1 \mathrm{~A}$.

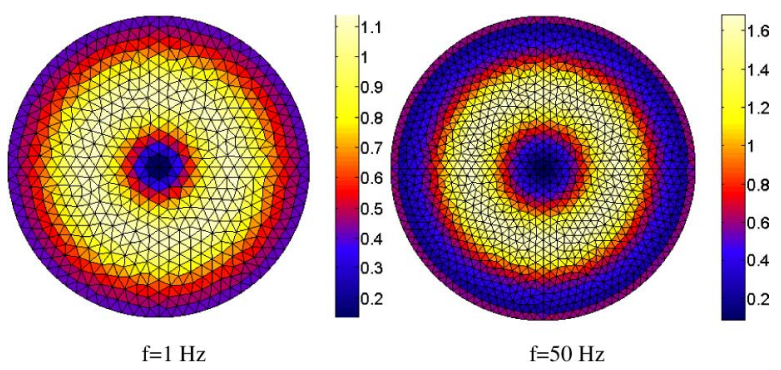

Fig. 7. Surface distribution of current $(\mathrm{A} / \mathrm{m})$ in the disk.

$\mathbf{H}_{0}$ created by a loop fed with $\mathrm{I}=1$ A (Fig. 6). This example is modeled by three different methods. The first one is the axisymmetric FEM. The second one is a shell element formulation implemented in 3-D FEM code [7], [10]. The last one is the considered integral method. We still focus on the computed eddy current distribution and Joule losses in the disk at different frequencies (see Table II, Figs. 7 and 8).

If we consider that axisymmetric FEM method as our reference, the integral method leads to an error of $2.7 \%$ for the Joule losses at $\mathrm{f}=50 \mathrm{~Hz}$ (Fig. 8). The lower the skin depth is, the lower the error is. These errors can be explained by the modeling of the geometry with a strong edge effect where the infinite plane assumption leads to inaccuracies. Let us notice that our integral formulation seems to lead to more accurate results than the same implementation of the shell element but with the air region treated with a 3-D FEM. Maybe a more precise nu-

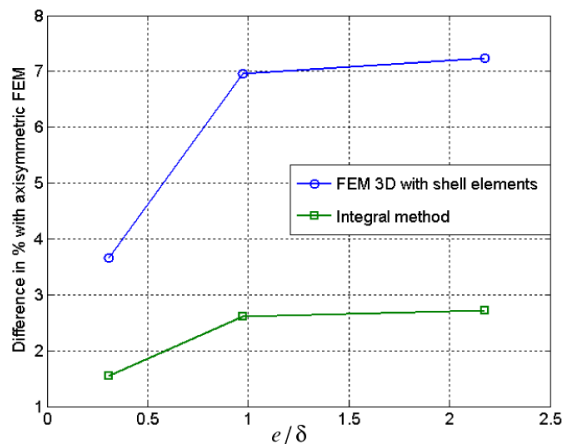

Fig. 8. Difference in $\%$ with the axisymmetric FEM solution for the Joule losses computation versus $\mathrm{e} / \delta$.

TABLE II

JOULE LOSSES OF THE DISK (W)

\begin{tabular}{|c|c|c|c|c|}
\hline $\mathrm{f}(\mathrm{Hz})$ & $\mathrm{e} / \delta$ & $\begin{array}{c}\text { Axisymmetric } \\
\text { FEM method }\end{array}$ & $\begin{array}{c}\text { Our } \\
\text { method }\end{array}$ & $\begin{array}{c}\text { FEM 3D } \\
\text { with shell } \\
\text { elements }\end{array}$ \\
\hline 1 & 0.30 & $7.10 \mathrm{E}-7$ & $7.31 \mathrm{E}-7$ & $6.84 \mathrm{E}-7$ \\
\hline 10 & 0.97 & $1.15 \mathrm{E}-6$ & $1.18 \mathrm{E}-6$ & $1.07 \mathrm{E}-6$ \\
\hline 50 & 2.17 & $2.21 \mathrm{E}-6$ & $2.26 \mathrm{E}-6$ & $2.05 \mathrm{E}-5$ \\
\hline
\end{tabular}

merical study should be made in order to better understand this difference.

\section{CONCLUSION}

In this paper, we have presented an original integral formulation using shell elements in order to model thin conductive nonmagnetic regions. The formulation is general and various skin effects across thickness $(\delta \gg e$ or $\delta \approx e$ or $\delta \ll e)$ are taken into account. The coupling of this formulation with compression algorithm seems to be promising in order to model real shielding applications. In further work, similar formulations enabling the modeling of thin conductive magnetic shells will be focused.

\section{REFERENCES}

[1] S. J. Salon, B. Mathewson, and S. Uda, "An intergro-differential approach to eddy currents in thin plates," IEEE Trans. Magn., vol. MAG-19, no. 6, pp. 2405-2408, 1983.

[2] H. Tsuboi, M. Tanaka, and T. Misaki, "Eddy current and deflection analyses of a thin plate in time-changing magnetic field," IEEE Trans. Magn., vol. 26, no. 5, pp. 1647-1649, 1990.

[3] H. Chemkha and F. Rioux-Damidau, "A fast and accurate model for eddy current computation in thin conducting shells," IEEE Trans. Magn., vol. 33, no. 2, pp. 1342-1345, 1997.

[4] A. Canova, G. Gruosso, and M. Repetto, "Integral methods for analysis and design of low-frequency conductive shields," IEEE Trans. Magn., vol. 39, no. 4, pp. 2009-2017, 2003.

[5] S. Koch, J. Trommler, H. D. Gersem, and T. Weiland, "Modeling thin conductive sheets using shell elements in magnetoquasistatic field simulations," IEEE Trans. Magn., vol. 45, no. 2, pp. 1292-1295, 2009.

[6] L. Krähenbühl and D. Muller, "Thin layers in electrical engineering. Example of shell models in analyzing eddy-currents by boundary and finite element methods," IEEE Trans. Magn., vol. 29, no. 2, pp. 1450-1455, 1993.

[7] C. Guérin, G. Tanneau, G. Meunier, P. Labie, T. Ngnegueu, and M. Sacotte, "A shell element for computing 3D eddy current-Application to transformers," IEEE Trans. Magn., vol. 31, no. 3, pp. 1360-1363, 1995.

[8] A. Buchau, S. Tsafak, W. Hafla, and W. Rucker, "Parallelization of a fast multipole boundary element method with cluster openMP," IEEE Trans. Magn., vol. 44, no. 6, pp. 1338-1341, 2008.

[9] R. Banucu, J. Albert, C. Scheiblich, V. Reinauer, W. Rucker, A. Hafla, and A. Huf, "Design and optimization of a contactless magnetically levitated actuator for 4-axis-machining," IEEE Trans. Magn., vol. 47, no. 7, pp. 1050-1053, 2011.

[10] Flux, Cedrat. Meylan, France [Online]. Available: www.cedrat.com 